\title{
A Cost-Utility Analysis of Different Antiviral Medicine Regimens in Patients With Chronic Hepatitis C Virus Genotype 1 Infection
}

\author{
Seyed Moayed Alavian, ${ }^{1}$ Shekoufeh Nikfar, ${ }^{2}$ Abbas Kebriaeezadeh, ${ }^{2}$ Farhad Lotfi, ${ }^{3}$ Ehsan Sanati, ${ }^{2}$ \\ Mohsen Rezaei Hemami, ${ }^{4}$ and Khosro Keshavarz ${ }^{3,}{ }^{*}$ \\ ${ }^{1}$ Baqiyatallah Research Center for Gastroenterology and Liver Diseases, Baqiyatallah University of Medical Sciences, Tehran, IR Iran \\ ${ }^{2}$ Department of Pharmacoeconomics and Pharmaceutical Administration, Faculty of Pharmacy, Tehran University of Medical Sciences, Tehran, IR Iran \\ ${ }^{3}$ Health Human Resource Research Center, School of Management and Information Sciences, Shiraz University of Medical Sciences, Shiraz, IR Iran \\ ${ }^{4}$ Institute of Health and Wellbeing, Health Economics and Health Technology Assessment, University of Glasgow \\ "Corresponding author: Khosro Keshavarz, Health Human Resource Research Center, School of Management and Information Sciences, Shiraz University of Medical Sciences, \\ Shiraz, IR Iran. Tel: +98-09173301430, E-mail: khkeshavarz2007@gmail.com
}

Received 2016 February 13; Revised 2016 June 05; Accepted 2016 August 14.

\begin{abstract}
Background: Despite the introduction of new drug regimens with high effectiveness for the hepatitis C virus (HCV) patients, especially in HCV genotype 1, no cost-effectiveness study on the selection of the superior drug strategy in Iran has been conducted yet.

Objectives: This study is aimed to assess the cost-effectiveness of the three drug regimens of pegylated interferon and ribavirin (PR), sofosbuvir (SOF) + PR and ledipasvir and sofosbuvir (LDV/SOF) in patients with HCV genotype 1 in Iran in the year 2014.

Methods: A Markov micro-simulation model was used to evaluate the cost-effectiveness of the three drug strategies for a cohort of 10000 patients. Quality-adjusted life-years (QALYs) were extracted from published studies. Cost data was estimated through the review of medical records and obtaining experts opinion.

Results: The results showed that the SOF + PR drug compared with PR had a lower cost and was more effective, but compared with the LDV/SOF, in spite of its lower cost, it was less efficient. The QALY values obtained for PR, SOF + PR and LDV/SOF, respectively, were $10.98,12.08$ and 12.28 and their costs were $\$ 41,741, \$ 7,676$ and $\$ 46,993$. Moreover, the results obtained from acceptability curves showed that SOF + PR were the most cost-effective treatment for thresholds below \$ 45,270 PPP.

Conclusions: The use of SOF + PR regimen or LDV/SOF can significantly reduce the incidence of complications associated with the disease. For example, short and long-term outcomes are better than the current drug regimens for HCV genotype 1 patients in all stages of the disease.
\end{abstract}

Keywords: Chronic Hepatitis C, Cost-Utility Analysis, Markov Model

\section{Background}

Hepatitis C can incessantly have a substantial impact on the health system with an increasing burden being expected in the next decade due to related mortality, morbidity, and liver transplantation (1). Early treatments of chronic HCV can considerably decrease the risk of developing advanced liver disease such as cirrhosis and liver cancer, decrease mortality and thus reduce the related costs (2-4).

Treatment of chronic HCV infection, with PR as the previous standard of care, is lengthy (24 to 48 weeks) and toxic with moderate efficacy. This treatment can result in sustained virological response (SVR) in about $80 \%$ of people with genotypes 2 and 3 and about $40 \%-50 \%$ of people with genotype $1(5,6)$.
However, the treatment of chronic HCV infection is rapidly improving (7-11). The new regimens are highly efficacious ( $>90 \%$ SVR), have reduced the duration of treatment ( 8 to 12 weeks) and have minimal side effects (12), which resulted in improving the patients' quality of life (6). The high price of the new regimens is the main factor limiting their use in many countries.

It is estimated that about 187,000 people with chronic HCV infection live in Iran with more than $50 \%$ of them being infected with genotype 1 (13).

Since SOF is a new drug, there are not many economic evaluation studies on the drug throughout the world. However, the results of a few conducted studies suggests that compared with current treatments for HCV genotype 1 , the SOF + PR or LDV/SOF drug regimens can be associated with more favorable short-term and long-term health and 
economic outcomes at all levels and stages of the disease and they can be considered as a more cost effective option, especially for genotype $1(14,15)$. Several economic evaluation studies on the treatment of $\mathrm{CHC}$ have been conducted and published in America and Europe. However, in the Eastern Mediterranean region (EMRO), particularly Iran, there is no published study using economic evaluation models for the study of drug therapy protocols in patients with hepatitis $\mathrm{C}$. Thus, because of the lower rate of improvement in patients with HCV genotype 1 , it is necessary to carry out an economic evaluation study to determine the most cost effective strategy amongst the three strategies of PR, SOF+ PR and SOF/LDV. Therefore, this study is aimed to assess the cost-utility of patients with HCV genotype 1 from the perspective of payers and determine the most appropriate drug treatment protocols for this group of patients with regards to cost-utility and economic considerations in the country.

\section{Objectives}

This study is aimed to assess the cost-effectiveness of three drug regimens of pegylated interferon and ribavirin (PR), sofosbuvir (SOF) + PR and ledipasvir and sofosbuvir (LDV/SOF) in patients with HCV genotype 1 in Iran in the year 2014 .

\section{Methods}

\subsection{Model Overview}

In this study, we conducted a cost-utility analysis of three drug therapy strategies including PR, SOF + PR and SOF/LDV, which are used for patients with HCV genotype 1. Due to the chronic and recursive nature of HCV infection, the analysis was carried out using the Markov model. The outcome used in this model included QALYs, the costs spent on each health state and each treatment strategy. The scope of the study was the payer; therefore, direct medical and non-medical costs were investigated. The time horizon of the study was the patients' lifetime and the time interval was one year. For every one year time interval in the model the patients remained at their current state or moved to other health states such as SVR with compensated cirrhosis, SVR without CC, compensated cirrhosis (CC), decompensated cirrhosis (DC), hepatocellular carcinoma (HCC), liver transplant and death. For the nature of changes in disease states to be consistent with other published cost-utility studies (14-17) and to follow the opinions of experts, the time interval was selected to be one-year cycle. Since the time horizon was more than one year, the economic and clinical outcomes were discounted with an annual rate of $7.2 \%(18)$ and $3 \%(19,20)$. The mentioned discount rates have been previously used by other published economic evaluation studies in Iran (20). The analysis was carried out using Tree Age 2011 software.

\subsection{Patient Population}

Because of the lack of evidence in the country regarding the two regimens SOF+PR and SOF/LDV, we used information including effectiveness and transition probability taken from studies published in other countries. However, data on patients using PR was collected from 144 profiles registered by the research center of gastroenterology and hepatology in Baqiyatallah University. Approximately $78 \%$ of these patients did not have cirrhosis (score F0- F3) and $22 \%$ did (score F4) all with a mean age of 50 years. This is consistent with the reported proportion of HCV patients with cirrhosis in Iran, which is approximately $20 \%$ (21). These centers are national referral centers and patients from all over the country are admitted to there for treatment. Micro-simulation analysis was carried out for a cohort hypothetical population of 10000 people.

Patients who were infected with $\mathrm{CHC}$ and diagnosed by biopsy or Fibro Sca were entered into the study.

\subsection{Switching Drugs}

In this study, if patients had no response, showed drug resistance or showed side effects due to initial drug therapy regimens their drugs were switched. Nowadays, combinations of PR regimens are used for treating patients with HCV genotype 1 . According to the published studies, in this group of patients, the response rate to this drug regimen is only $40 \%-50 \%(5,22)$, which requires the patients who do not respond to the treatment to use a new drug regimen. Recently, two drug regimens, i.e. SOF + PR and SOF/LDV, have been introduced in the country and according to the published evidence the response rate for these drugs in patients with HCV genotype 1 is more than $90 \%$ $(23,24)$. Therefore, for patients who had no response to the current treatment regimen, a switch to a new drug regimen was used and a new state called withdrawal was added to the model.

\subsection{Model Structure}

Figure 1 shows a schematic diagram of the Markov model for a simulated population of $10000 \mathrm{CHC}$ patients. As indicated by the diagram, $\mathrm{CHC}$ patients who are taking the drug can undergo a transition in a reciprocating state. The intended states and their changes were determined and utilized based on the evidence found in the published studies and the opinions of experts. Patients with different health states and are undergoing treatment can face HCC 
and death. Those with chronic hepatitis $\mathrm{C}$ infection were entered into the Markov model as the initial distribution.

The designed Markov model compared the three drug therapy strategies and the patients who were evaluated by the model received one of the three drug regimens (see Figure 1).

\subsection{Model Assumptions}

- According to the available clinical evidence, the response rate for SOF + PR and SOF/LDV is assumed to be $100 \%$.

- The SVR state in the model is assumed as the goal of treatment and as the cure state.

- It is assumed that patients who reach the SVR state in METAVIR fibrosis score F0 - F2 are left in this state and do not experience the progress of the disease until death.

- It is assumed that patients who reach the SVR state in METAVIR fibrosis score F3 - F4 are possibly less exposed to the risk of developing liver disease than those who do not reach SVR state.

- It is assumed that patients who do not reach the SVR state are at risk of the progress of liver disease.

- The probabilities for some transitions were only available for some drugs. In such cases, regardless of the drug, it was assumed that all treated patients had equal chances of improvement or progression of the disease.

\subsection{Transitional Probabilities}

Transitional probabilities of parameters used in the model are presented in Table 1 . Due to the lack of evidence in the country, especially clinical data about the new drug regimens administered for patients with chronic hepatitis $\mathrm{C}$, most of the transitional probabilities of different health states were extracted from the published studies which were solely focusing on this issue $(4,25-32)$.

To include the cause of death other than HCV, we used the probabilities of life tabulation 2012 by age and sex. The probabilities of HCV related deaths were extracted from the published studies $(4,14,15,25-32)$. Some other probabilities were also extracted through assessing the registry of patients in the studied centers.

As a number of articles had reported the data on clinical efficacy by rate, we first changed the rates into probabilities using the following formula (33) and then included them in the model.

Equation 1.

$P=1-e x p^{(-r t)}$

Where P, probability; r, rate; t, time.

\subsection{Health Outcomes}

In this study, QALY was considered as the health outcome. The utility values used in the model are presented in Table 1. Since the two drug regimens of SOF + PR and SOF/LDV are recently introduced and used in the country, there is a lack of sufficient evidence determining the values used in the model, we used the evidence found in studies published in other countries (14-17, 34).

\subsection{Cost Inputs}

The data on direct medical costs on different states of $\mathrm{CHC}$ and other complications associated with the disease such as CC, DC and HCC were extracted from outpatient and inpatient medical records in the mentioned treatment centers and the self-reports of specialists. For each disease state there was an attempt to collect data on direct medical costs including the average number of physician visits, prescribed medicines, diagnostic services, treatment and hospitalization and use of other services.

Resources used to calculate the costs were based on prices from 2014. To make international comparisons we tried to convert the costs to international dollars using the purchasing power parity (PPP) with an exchange rate of 8565.41 Rials per \$ 1 (35). The cost of each health state is presented separately in Table 1 with the cost details shown in Table 2.

\subsection{Cost-Effectiveness Analysis}

Based on the results obtained from the previous stages, a Markov model was applied using the Tree age software and the collected data was included into the model. Taking into account the time horizon of the study, we calculated the costs, effectiveness and cost-effectiveness for all interventions with monetary units. QALY, Cost per QALY and their ICERs were obtained using the following formula (36).

$I C E R=\frac{\text { Cost } A-\operatorname{Cost} B}{\text { OutcomeA }- \text { OutcomeB }}$

Where A, SOF/LDV; B, other antiviral.

\subsection{Sensitivity Analysis}

To measure the level of certainty in base-case results, a probabilistic sensitivity analysis (PSA) was performed on the results of the model. Therefore, with regards to the defined probability distributions for the parameters that were entered into the model, a second-order Monte Carlo simulation model was conducted using 5000 trials to analyze the probabilistic sensitivity. PSA results are presented using the cost-effectiveness acceptability curves and incremental cost-effectiveness scatter plot. In this article, the 
Table 1. Input Parameters Used in Economic Model: HCV

\begin{tabular}{|c|c|c|c|c|c|}
\hline Variable & Mean & Distribution & SD & Alpha & Beta \\
\hline Prob CHC to W_pr & 0.55 & Beta & 0.2258 & 2.67 & 2.18 \\
\hline Prob CHC to SVR_pr & 0.45 & Beta & 0.2258 & 2.18 & 2.67 \\
\hline Prob CHC to CC & 0.115 & Beta & 0.04106 & 6.94 & 53.43 \\
\hline Prob CHC to HCC & 0.011 & Beta & 0.04106 & 0.07 & 6.38 \\
\hline Prob CC to $W_{-}$pr & 0.55 & Beta & 0.23868 & 2.39 & 1.96 \\
\hline Prob CC to SVR_pr & 0.33 & Beta & 0.23868 & 1.28 & 2.60 \\
\hline Prob CC to DC & 0.039 & Beta & 0.04106 & 0.87 & 21.36 \\
\hline Prob CC to HCC & 0.024 & Beta & 0.04106 & 0.33 & 13.56 \\
\hline Prob DC to LT & 0.031 & Beta & 0.04106 & 0.55 & 17.27 \\
\hline Prob LT to Death & 0.107 & Beta & 0.04106 & 6.06 & 50.61 \\
\hline Prob DC to HCC & 0.014 & Beta & 0.04106 & 0.11 & 8.07 \\
\hline Prob CHC to SVR_Is & 0.977 & Beta & 0.2258 & 0.43 & 0.01 \\
\hline Prob CC to SVR_ls & 0.96 & Beta & 0.23868 & 0.65 & 0.03 \\
\hline Prob CHC to SVR_spr & 0.91 & Beta & 0.2258 & 1.46 & 0.14 \\
\hline Prob CC to SVR_spr & 0.79 & Beta & 0.23868 & 2.30 & 0.61 \\
\hline Utility CHC_pr & 0.6657 & Beta & 0.085 & 20.50 & 10.30 \\
\hline Utility CHC_spr & 0.645 & Beta & 0.085 & 20.44 & 11.25 \\
\hline Utility CHC_sl & 0.835 & Beta & 0.085 & 15.92 & 3.15 \\
\hline Utility CC_pr & 0.6257 & Beta & 0.085 & 20.28 & 12.13 \\
\hline Utility CC_spr & 0.605 & Beta & 0.085 & 20.01 & 13.07 \\
\hline Utility CC_sl & 0.795 & Beta & 0.085 & 17.93 & 4.62 \\
\hline Utility DC & 0.525 & Beta & 0.08957 & 16.32 & 14.76 \\
\hline Utility DC_spr & 0.525 & Beta & 0.08957 & 16.32 & 14.76 \\
\hline Utility DC_sl & 0.715 & Beta & 0.08957 & 18.16 & 7.24 \\
\hline Utility HCC & 0.465 & Beta & 0.08957 & 14.42 & 16.59 \\
\hline Utility HCC_spr & 0.465 & Beta & 0.08957 & 14.42 & 16.59 \\
\hline Utility HCC_sl & 0.655 & Beta & 0.08957 & 18.45 & 9.72 \\
\hline Cost CHC_sl & 86208 & Gamma & 34152 & 6.37 & 0.00 \\
\hline Cost CHC_spr & 11903 & Gamma & 34152 & 0.12 & 0.00 \\
\hline Cost CHC_pr & 15772 & Gamma & 34152 & 0.21 & 0.00 \\
\hline Cost CC_sl & 86664 & Gamma & 34035 & 6.48 & 0.00 \\
\hline Cost CC_spr & 12359 & Gamma & 34035 & 0.13 & 0.00 \\
\hline Cost $\mathrm{CC}_{-} \mathbf{p r}$ & 16771 & Gamma & 34035 & 0.24 & 0.00 \\
\hline Cost DC_sl & 101124 & Gamma & 33796 & 8.95 & 0.00 \\
\hline Cost DC_spr & 36862 & Gamma & 33796 & 1.19 & 0.00 \\
\hline Cost DC_pr & 23798 & Gamma & 33796 & 0.50 & 0.00 \\
\hline Cost HCC_sl & 112023 & Gamma & 31208 & 12.88 & 0.00 \\
\hline Cost HCC_spr & 52508 & Gamma & 31208 & 2.83 & 0.00 \\
\hline Cost HCC_pr & 40718 & Gamma & 31208 & 1.70 & 0.00 \\
\hline Prob HCC to LT & 0.0003 & Beta & 0.04106 & 0.00 & 0.18 \\
\hline Prob CHC to SVR_pr_spr & 0.72 & Beta & 0.2258 & 2.85 & 1.11 \\
\hline Prob CC to SVR_pr_spr & 0.62 & Beta & 0.23868 & 2.56 & 1.57 \\
\hline Prob CHC to SVR_pr_Is & 0.79 & Beta & 0.2258 & 2.57 & 0.68 \\
\hline Prob CC to SVR_pr_ls & 0.8 & Beta & 0.23868 & 2.25 & 0.56 \\
\hline
\end{tabular}

Abbreviations: Prob, probability; CC, compensated cirrhosis; DC, decompensated cirrhosis; HCC, hepatocellular carcinoma; SVR, sustained virological response; LT, liver transplantation; W, withdrawal; pr, pegylated interferon and ribavirin; spr, sofosbuvir + pegylated interferon and ribavirin; sl, ledipasvir and sofosbuvir; SD, standard deviation, Alpha and Beta are the distribution kind of mentioned variables.

PSA results are characterized with the use of incremental cost-effectiveness, scatter plot and cost-effectiveness acceptability curves. Due to the lack of WTP threshold in Iran the WTP per every QALY, based on the WHO's approach was considered as one to three times of gross domestic product per capita. In 2014, it was 4670 dollars per capita (37). Meanwhile, due to international comparison, we used the latest gross domestic product per capita based on the pur- 
Table 2. Results of Drug Acquisition Costs Used in the Model

\begin{tabular}{lc}
\hline Treatment & Unit cost, PPP S (Per pill) \\
\hline Ribavirin & 0.7 \\
Pegylated interferon & 173 \\
\hline Sofosbuvir & 60 \\
\hline Ledipasvir/sofosbuvir & 979 \\
\hline
\end{tabular}

chasing power parity (PPP), which was 15090.05 US dollars in $2013(37,38)$.

\section{Results}

In this study, based on the initial distribution of the model, a total of $144 \mathrm{CHC}$ patients who were 14 years of age and were referred to the selected centers were enrolled in the study and were classified by age, sex and duration of the disease. The mean age of the patients was 50 years; with $79 \%$ being male and $21 \%$ female.

The results showed that SOF + PR drug, compared with the two drug therapy strategies, was more cost effective. When compared to the PR it had a lower cost and was more effective but compared with the LDV/SOF, in spite of its lower cost, it was less efficient. As a whole, of the three above-mentioned strategies, SOF + PR was the most cost effective strategy with the next being LDV/SOF. PR drug, which had the highest cost and was less effective than the other strategies, was the failed strategy (see Figure 2, Table 3).

Cost, effectiveness, incremental costs, incremental effectiveness, ICER as well as ratings were the superiority of the three drug strategies in $\mathrm{CHC}$ patients. The comparison is presented in Table 3. The findings shown in the tabulation indicate that the QALYs for each of the three medicines of PR, SOF + PR, LDV/SOF were 10.98, 12.08 and 12.28; in addition, the costs were \$41.741, \$ 7.676 and \$ 46.993 As shown and compared with other alternatives, PR and LDV/SOF had the minimum and maximum expected effectiveness and SOF + PR and LDV/SOF had the minimum and maximum expected costs over the period of a life time. Furthermore, LDV/SOF with about 190,335 dollars PPP is the highest cost per QALY compared with the other strategies (Table 3).

\subsection{Probabilistic Sensitivity Analysis (PSA)}

In this study, because it was possible to measure all the variables, they were considered to be distributed. In this study, the distribution of beta $(\beta)$ was used to determine the distribution of transitional probabilities and utility values that were between 0 to 1 . Additionally the gamma distribution was used to determine the distribution of the costs (Table 1). Thus, a second-order Monte Carlo simulation using 5000 trials was conducted for the analysis of probabilistic sensitivity. The results of the measurement of uncertainty using cost-effectiveness acceptability curves and incremental cost-effectiveness scatter plot (ICER) distribution curves are as follows.

The results of the acceptability curves showed that in 91.7\% to $85.7 \%$ of simulations SOF + PR was the most cost effective treatment for thresholds less than 45,270 US dollars. The next best drug strategy was LDV/SOF which was the optimal strategy in $4.4 \%$ to $12.5 \%$ of the simulations. PR drug was the best strategy only in $3.9 \%$ to $1.8 \%$ of the simulations (see Figure 3). Moreover, the results of the bar graph showed that in $85.7 \%$ of simulations SOF + PR drug in thresholds less than $\$ 45,270$ was identified as the best strategy while the other strategies were superior strategies only in $14.3 \%$ of simulations (see Figure $4 \mathrm{~A}-4 \mathrm{~B}$ ).

The scatter plots provide more detailed information for individual comparisons. The results of scatter plots are only shown for the two superior options compared with PR. The results of the scatter plots showed that in 92\% and $32 \%$ of simulations, respectively, SOF + PR and LDV/SOF, were located in the fourth region of the cost-effectiveness plot. For example in a region with lower-costs and higher effectiveness, as compared with PR, they were considered as the superior strategies. In $2 \%$ and $58 \%$ of simulations, they were in the first region, under the threshold. For example in a region with higher costs and higher effectiveness, compared with the PR. In $2 \%$ and $0 \%$ of the simulations they were under the threshold at the third region. Therefore, overall SOF + PR and LDV/SOF in 96\% and 90\% of simulations were at the acceptable region, below the threshold, as compared with the PR and as a result they defeated the PR strategy and were identified as the more cost effective strategies. Moreover, SOF + PR and LDV/SOF in 4\% and $10 \%$ of the simulations were in the rejected area above the threshold as compared with the PR and were identified as the non-cost effective (inferior) strategies.

Therefore, the results of sensitivity analysis showed that SOF + PR was the most effective treatment and LDV/SOF was the subsequent treatment strategy with no change in their status compared with the PR strategy. This indicates that the results of this study are robust.

The results also showed that given the better effectiveness of LDV/SOF drug strategy, if the price of the drug decreases from its current price, i.e. from around 980 dollars PPP to 120 dollars PPP per each pill, then it will become the most cost effective strategy and LDV/SOF drug will become dominant and the other two drug strategies of SOF + PR and PR will be totally dominated (see Figure 5). As the results of acceptability curves obtained from the sensitivity analysis showed, in $50 \%$ to $89.7 \%$ of simulations, LDV/SOF 
Table 3. The Results of a Markov Micro-Simulation Model to Compare the Cost-Effectiveness of the Three Drug Strategies in Patients with CHC Based on the Number QALY (USD)

\begin{tabular}{|c|c|c|c|c|c|c|c|}
\hline Strategy & Cost & Eff(QALY) & Inc Cost & Inc Eff & Inc CE & Rank & Subset \\
\hline SOF + PR & 7,676 & 12.08 & 0 & 0 & 0 & 1 & Dominant \\
\hline SOF + LDV & 46,993 & 12.28 & 39,316 & 0.21 & 190335 & 2 & Dominant \\
\hline PR & 41,741 & 10.98 & 34,064 & -1.10 & -30985 & 3 & abs. dominated \\
\hline
\end{tabular}

Abbreviations: Eff, Effectiveness; Inc, incremental cost; Inc Eff, incremental effectiveness, Inc CE, incremental cost-effectiveness; PR, pegylated interferon/ribavirin; SOF + PR, sofosbuvir + pegylated interferon/ribavirin; SOF + LDV, ledipasvir + sofosbuvir; CHC, chronic hepatitis C; QALY, quality-adjusted life-year.

was the most cost effective treatment for thresholds between 4527 to 45270 dollars PPP. SOF + PR drug was optimal only when it was below the threshold of 4527 dollars PPP (see Figure 6).

Figure 1. Decision Analysis Model to Evaluate the Cost-Effectiveness of Treatment Strategies for Chronic HCV Patients

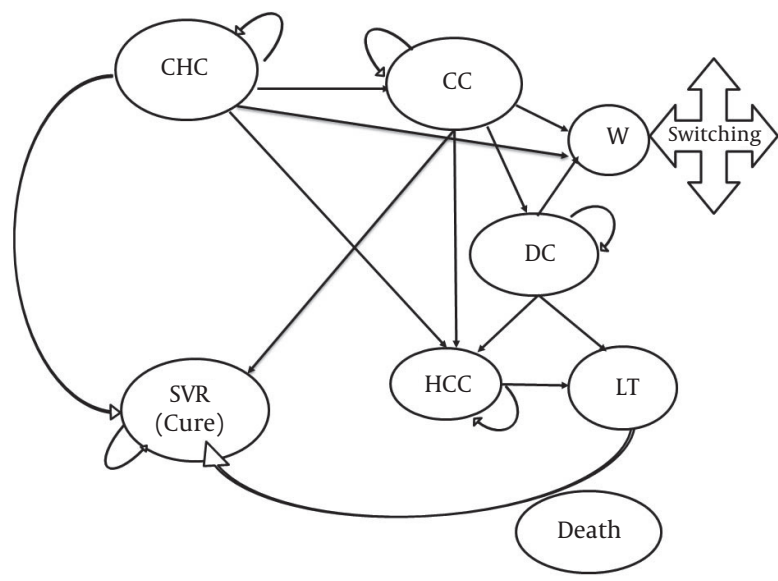

CHC, chronic hepatitis C; CC, compensated cirrhosis; DC, decompensated cirrhosis; HCC, hepatocellular carcinoma; SVR, sustained virological response; LT, liver transplantation; $\mathrm{W}$, withdrawal.

\section{Discussion}

Since LDV/SOF medicine is a new drug, there are a limited number of economic evaluation studies published in the world on the drug. Hence, this study was the first economic evaluation research on hepatitis $C$ patients in the country and in the EMRO region. Furthermore, it was one of the very few studies conducted in the world, which tried to take a pharmacoeconomic view to assess the three different drug therapy options for patients with HCV genotype 1 . Therefore, this study was carried out to fulfill one of the goals of policy decision makers to select the best drug strategies for the inclusion in the Iran drug list(IDL). In this study, based on the characteristics of the sample under the study analysis, micro-simulation analysis was conducted on CHC patients in a population of 100 thousand people.
The results showed that compared with other alternatives and considering the given prices, SOF + PR was the most cost-effective strategy. This strategy will have the most favorable health economic outcomes in the future compared with other strategies available for patients with $\mathrm{HCV}$ and its related complications. In comparison with the SOF + PR, although LDV/SOF drug regimen has the highest rate of SVR, it is not a treatment priority due to its very high price in the country. Therefore, under a fixed budget, more patients can successfully be treated by the SOF + PR drug regimen as compared with other treatment alternatives. In view of that, the results of this study are in line with those of Saab et al.'s study (15). Therefore, SOF + PR has a lower cost and is more effective than PR. Our results are consistent with the findings of the recently published studies, indicating that the SOF drug regimen, compared with the other current standard drug regimens, is more effective for the treatment of HCV genotype $1(14,15)$. Also, the results of the probability sensitivity analysis showed that the SOF + PR drug regimen, compared with the two other medication strategies was more cost effective and in most simulations the obtained values were at the accepted area and below the threshold; thus, it obtained the best results with respect to the given prices. Therefore, the results showed that the sensitivity analysis did not change the status of SOF + PR as the most effective drug regimen; this indicates that the results of this study are robust. Accordingly, the results of this study are in line with those of Saab et al.'s study in the USA and Petta et al.'s study in Italy $(15,16)$.

The second generation of direct-acting anti-viral drugs (all-oral) such as LDV/SOF has a high efficacy and safety. It is also a simple treatment strategy that is free of PR and makes great progress in improving the patients during treatment and significantly increases SVR rate (39-41). Therefore, the results showed that if the price of this drug reduces, it will be the most cost effective strategy and completely dominates the other two drug regimens of SOF+PR and PR. As the results of acceptability curves are obtained from the sensitivity analysis indicated, LDV/SOF with its new prices could be the most cost effective treatment in 89.7\% of simulations for thresholds below 45270 dollars PPP.

Thus, our results showed that SOF + PR or LDV/SOF drug 


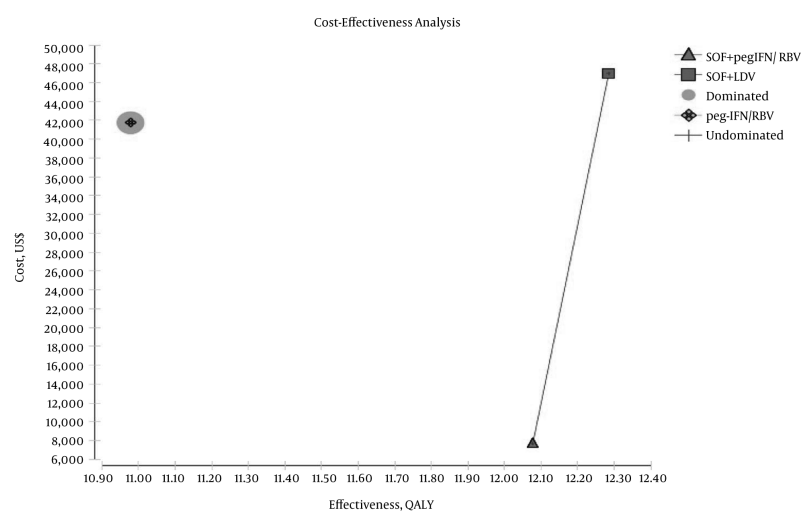

Peg-IFN/RBV, pegylated interferon/ribavirin; SOF + Peg-IFN/RBV, sofosbuvir + pegylated interferon/ribavirin; SOF + LDV: ledipasvir + sofosbuvir; QALY, quality-adjusted life-year.

Figure 3. Cost-Effectiveness Acceptability Curve Obtained Through Monte Carlo Simulation

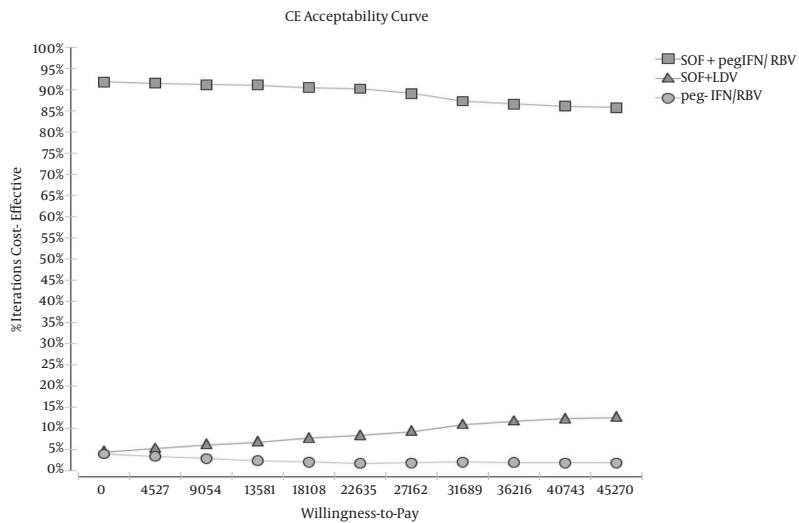

Peg-IFN/RBV, pegylated interferon/ribavirin; SOF + Peg-IFN/RBV, sofosbuvir + pegylated interferon / ribavirin; SOF + LDV, ledipasvir + sofosbuvir.

regimens could be associated with more favorable short and long term health economic outcomes, as compared with the current treatments for patients with HCV genotype 1 at all levels and stages of the disease. It can be a more cost effective option, especially for genotype 1 .

Therefore, in order to increase the rate of SVR, reduce the treatment period, increase the quality of life of patients and increase productivity during and after the treatment period on one hand and to significantly reduce the risk of complications associated with liver-disease, mortality and the costs for patients using SOF medication regimens $(6,14$, 15,42 ) on the other hand, it is recommended to stop the use of the current standard PR treatment regimen in Iran and many other countries, as they are not recommended for treating patients with HCV genotype 1. Therefore, a drug with high efficiency such as SOF should be used along with PR. Furthermore, as mentioned earlier, in the case of de- creasing the price of the drug (LDV/SOF), it will be better to use a PR-free drug regimen instead of the SOF + PR combination and use it as a priority for the treatment of patients. This PR-free regimen has shown high clinical efficacy, especially in patients with HCV genotype 1 who form the major number of HCV patients in Iran.

According to our study, to generalize the results to other settings it is better to consider some factors such as the availability of resources, prices, valuing outcomes by individuals, thresholds and other indicators of the effectiveness used in various studies that may affect the results of those studies. Therefore, the generalization of the results to other settings should proceed with caution.

One of the limitations of this study was the use of some data obtained from clinical trial studies which might differ slightly from real-world conditions. Another limitation was the lack of access to patients using SOF regimen, 
Incremental Cost-Effectiveness, SOF+pegIFN/RBV v.peg-IFN/RBV

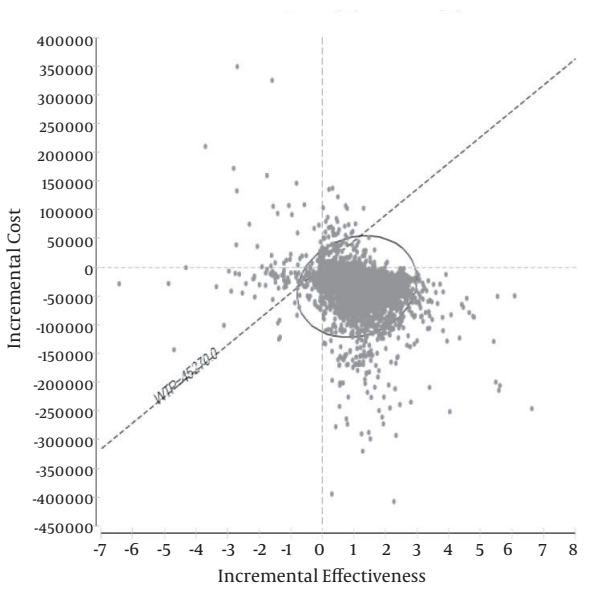

Incremental Cost-Effectiveness, SOF+LDV v.peg-IFN/RBV

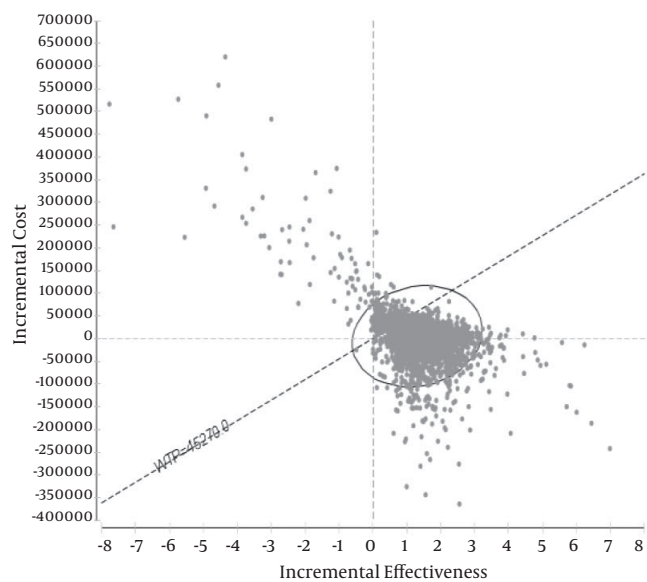

Peg-IFN/RBV, pegylated interferon/ribavirin; SOF + Peg-IFN/RBV, sofosbuvir + pegylated interferon/ribavirin; SOF+LDV, ledipasvir + sofosbuvir.

Figure 5. Comparison of Cost-Effectiveness of the Three Drug Strategies in Patients With Chronic Hepatitis C Based on the Number of QALY
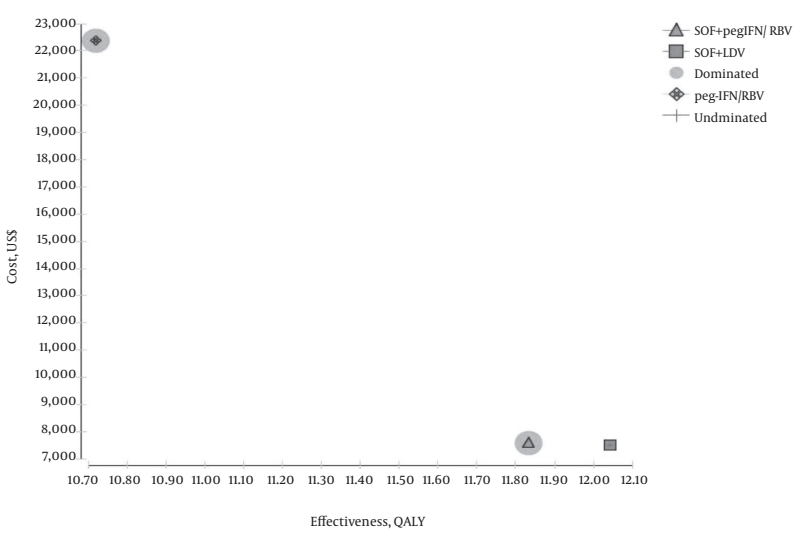

Effectiveness, QALY

Peg-IFN/RBV, pegylated interferon/ribavirin; SOF + Peg-IFN/RBV, sofosbuvir + pegylated interferon/ribavirin; SOF + LDV, ledipasvir + sofosbuvir; QALY, quality-adjusted life-year.

which was due to the high price of the drug in the country. Also, because of the limitations of the data required for some simulations we had to use the constant of transitional probabilities in the model in this study.

In conclusion, based on the findings of this study, it can be concluded that the use of SOf + PR and PR-free drug regimens (LDV/SOF; Harvoni) in patients with HCV genotype 1 can reduce the side effects associated with the disease such as HCC, DC and mortality. Additionally, it can reduce the costs and time spent on the disease, especially if the patient refers at early stages of the disease without cirrhosis and without previous experience of therapy. Even in treatment-experienced and cirrhosis patients, the men- tioned treatments are the most cost-effective option in the current situation and can be used as top priority drugs. They can also be used for switching from PR to the mentioned drugs.

\section{Acknowledgments}

The authors would like to thank Baqiyatallah research center for gastroenterology and liver diseases, Shiraz University of Medical Sciences, Shiraz, Iran, Center for development of clinical research of Nemazee hospital as well as Dr. Nasrin Shokrpour for editorial assistance. 


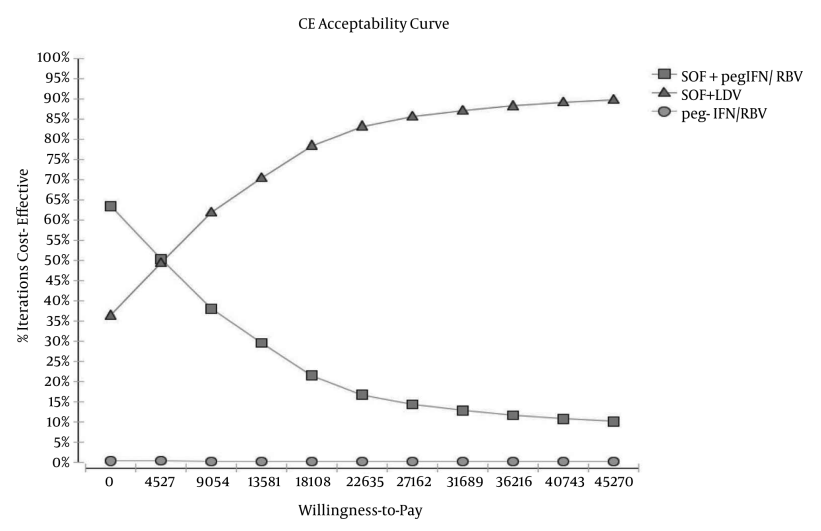

Peg-IFN/RBV, pegylated interferon/ribavirin; SOF + Peg-IFN/RBV, sofosbuvir + pegylated interferon/ribavirin; SOF + LDV, ledipasvir + sofosbuvir .

\section{Footnotes}

Authors' Contribution: Study concept and design, acquisition of data, analysis and interpretation of data, drafting of the manuscript, critical revision of the manuscript for important intellectual content and statistical analysis, Khosro Keshavarz; drafting of the manuscript, administrative, technical, and material support, and study supervision, Seyed Moayed Alavian, Shekoufeh Nikfar and Abbas Kebriaeezadeh; drafting of the manuscript and statistical analysis, Farhad Lotfi, Ehsan Sanati, and Mohsen Rezaei Hemami; All authors read and approved the final manuscript.

Funding/Support: The study was supported by Baqiyatallah research center for gastroenterology and liver diseases, Baqiyatallah University of Medical Sciences, Tehran, IR Iran.

\section{References}

1. Alavian SM. Hepatitis C virus infection: Epidemiology, risk factors and prevention strategies in public health in IR IRAN. Gastroenterology and Hepatology from bed to bench. 2009;3(1).

2. Gordon SC, Hamzeh FM, Pockros PJ, Hoop RS, Buikema AR, Korner EJ, et al. Hepatitis $C$ virus therapy is associated with lower health care costs not only in noncirrhotic patients but also in patients with end-stage liver disease. Aliment Pharmacol Ther. 2013;38(7):784-93. doi: 10.1111/apt.12454. [PubMed: 23981040].

3. van der Meer AJ, Veldt BJ, Feld JJ, Wedemeyer H, Dufour JF, Lammert $\mathrm{F}$, et al. Association between sustained virological response and all-cause mortality among patients with chronic hepatitis $\mathrm{C}$ and advanced hepatic fibrosis. JAMA. 2012;308(24):2584-93. doi: 10.1001/jama.2012.144878. [PubMed: 23268517].

4. D’Ambrosio R, Aghemo A, Rumi MG, Ronchi G, Donato MF, Paradis $\mathrm{V}$, et al. A morphometric and immunohistochemical study to assess the benefit of a sustained virological response in hepatitis $C$ virus patients with cirrhosis. Hepatology. 2012;56(2):532-43. doi: 10.1002/hep.25606. [PubMed: 22271347].
5. Ashtari S, Vahedi M, Pourhoseingholi MA, Karkhane M, Kimiia Z, Pourhoseingholi A, et al. Direct medical care costs associated with patients diagnosed with chronic HCV. Hepat Mon. 2013;13(5):8415. doi: 10.5812/hepatmon.8415. [PubMed: 23930132].

6. Pourhoseingholi MA, Ashtari S, Alavian SM. Sofosbuvir vs. Combination of Pegylated Interferon and Ribavirin; How Much Shall Pay for Iranian Patients?. Hepat Mon. 2014;14(11):25540. doi: 10.5812/hepatmon.25540. [PubMed: 25598793].

7. Liang TJ, Ghany MG. Therapy of hepatitis C-back to the future. $N$ Engl J Med. 2014;370(21):2043-7. doi:10.1056/NEJMe1403619. [PubMed: 24795199].

8. Sheridan C. FDA approvals usher in the post-interferon era in HCV. Nat Biotechnol. 2014;32(1):3-5. doi: 10.1038/nbt0114-3. [PubMed: 24406908].

9. Au JS, Pockros PJ. Novel therapeutic approaches for hepatitis C. Clin Pharmacol Ther. 2014;95(1):78-88. doi: 10.1038/clpt.2013.206. [PubMed: 24126682].

10. Afdhal N, Zeuzem S, Kwo P, Chojkier M, Gitlin N, Puoti M, et al. Ledipasvir and sofosbuvir for untreated HCV genotype 1 infection. N Engl J Med. 2014;370(20):1889-98. doi: 10.1056/NEJMoa1402454. [PubMed: 24725239].

11. Kowdley KV, Gordon SC, Reddy KR, Rossaro L, Bernstein DE, Lawitz E, et al. Ledipasvir and sofosbuvir for 8 or 12 weeks for chronic HCV without cirrhosis. N Engl J Med. 2014;370(20):1879-88. doi: 10.1056/NEJMoa1402355. [PubMed: 24720702].

12. Lam AM, Murakami E, Espiritu C, Steuer HM, Niu C, Keilman $\mathrm{M}$, et al. PSI-7851, a pronucleotide of beta-D-2'-deoxy-2'-fluoro-2'-Cmethyluridine monophosphate, is a potent and pan-genotype inhibitor of hepatitis C virus replication. Antimicrob Agents Chemother. 2010;54(8):3187-96. doi:10.1128/AAC.00399-10. [PubMed: 20516278].

13. Keyvani H, Alizadeh AH, Alavian SM, Ranjbar M, Hatami S. Distribution frequency of hepatitis $C$ virus genotypes in 2231 patients in Iran. Hepatol Res. 2007;37(2):101-3. doi: 10.1111/j.1872-034X.2007.00015.x. [PubMed: 17300704].

14. Younossi ZM, Park H, Saab S, Ahmed A, Dieterich D, Gordon SC. Costeffectiveness of all-oral ledipasvir/sofosbuvir regimens in patients with chronic hepatitis $\mathrm{C}$ virus genotype 1 infection. Aliment Pharmacol Ther. 2015;41(6):544-63. doi: 10.1111/apt.13081. [PubMed: 25619871].

15. Saab S, Gordon SC, Park H, Sulkowski M, Ahmed A, Younossi Z. Cost-effectiveness analysis of sofosbuvir plus peginterferon/ribavirin in the treatment of chronic hepatitis $C$ virus genotype 1 infection. Aliment Pharmacol Ther. 2014;40(6):657-75. doi: 10.1111/apt.12871. [PubMed: 25065960]. 
16. Petta S, Cabibbo G, Enea M, Macaluso FS, Plaia A, Bruno R, et al. Cost-effectiveness of sofosbuvir-based triple therapy for untreated patients with genotype 1 chronic hepatitis C. Hepatology. 2014;59(5):1692-705. doi: 10.1002/hep.27010. [PubMed: 24691835].

17. Hagan LM, Sulkowski MS, Schinazi RF. Cost analysis of sofosbuvir/ribavirin versus sofosbuvir/simeprevir for genotype 1 hepatitis $\mathrm{C}$ virus in interferon-ineligible/intolerant individuals. Hepatology. 2014;60(1):37-45. doi: 10.1002/hep.27151. [PubMed: 24677184].

18. Abdoli G. Estimation of social discount rate for Iran. Eco Res Rev. 2009;10(3):135-56.

19. Robberstad B. Estimation of private and social time preferences for health in northern Tanzania. Soc Sci Med. 2005;61(7):1597-607. doi: 10.1016/j.socscimed.2005.03.013. [PubMed: 15885866].

20. Nikfar S, Kebriaeezadeh A, Dinarvand R, Abdollahi M, Sahraian MA, Henry D, et al. Cost-effectiveness of different interferon beta products for relapsing-remitting and secondary progressive multiple sclerosis: Decision analysis based on long-term clinical data and switchable treatments. Daru. 2013;21(1):50. doi: 10.1186/2008-2231-21-50. [PubMed: 23800250].

21. Alavian SM, Hajarizadeh B, Hajibeigi B, Doroudi T, Hamadanizadeh AK, Abar K. Efficacy and safety of pegylated interferon alfa-2a plus ribavirin for treatment of chronic hepatitis $\mathrm{C}$ and cirrhosis in Iranian. Hepat Mon. 2004;4(7):53-8.

22. Aval F, Behnaz N, Raoufy M, Alavian SM. Predicting the outcomes of combination therapy in patients with chronic hepatitis $\mathrm{C}$ using artificial neural network. Hepat mon. 2014;14(6).

23. Alavian SM. Sofosbuvir has come out of the magic box. Hepat mon. 2013;13(12).

24. Kowdley KV, Lawitz E, Crespo I, Hassanein T, Davis MN, DeMicco $\mathrm{M}$, et al. Sofosbuvir with pegylated interferon alfa-2a and ribavirin for treatment-naive patients with hepatitis $C$ genotype- 1 infection (ATOMIC): an open-label, randomised, multicentre phase 2 trial. Lancet. 2013;381(9883):2100-7. doi: 10.1016/S0140-6736(13)60247-0. [PubMed: 23499440].

25. Thein HH, Yi Q, Dore GJ, Krahn MD. Estimation of stage-specific fibrosis progression rates in chronic hepatitis $\mathrm{C}$ virus infection: a meta-analysis and meta-regression. Hepatology. 2008;48(2):418-31. doi: 10.1002/hep.22375. [PubMed: 18563841].

26. Dienstag JL, Ghany MG, Morgan TR, Di Bisceglie AM, Bonkovsky HL, Kim HY, et al. A prospective study of the rate of progression in compensated, histologically advanced chronic hepatitis C. Hepatology. 2011;54(2):396-405. doi: 10.1002/hep.24370. [PubMed: 21520194].

27. Morgan RL, Baack B, Smith BD, Yartel A, Pitasi M, Falck-Ytter Y. Eradication of hepatitis $C$ virus infection and the development of hepatocellular carcinoma: a meta-analysis of observational studies. Annals Inter Med. 2013;158(5_Part_1):329-37.

28. Fattovich G, Giustina G, Degos F, Diodati G, Tremolada F, Nevens $F$, et al. Effectiveness of interferon alfa on incidence of hepatocellular carcinoma and decompensation in cirrhosis type C. Hepatol. 1997;27(1):201-5.

29. Bennett WG, Inoue Y, Beck JR, Wong JB, Pauker SG, Davis GL. Estimates of the cost-effectiveness of a single course of interferon-alpha $2 \mathrm{~b}$ in patients with histologically mild chronic hepatitis C. Ann Intern Med. 1997;127(10):855-65. [PubMed: 9382363].

30. Liu J, Wang Y, Zhang D, Liu B, Ou Q. Comparison of survival and quality of life of hepatectomy and thrombectomy using total hepatic vascular exclusion and chemotherapy alone in patients with hepatocellular carcinoma and tumor thrombi in the inferior vena cava and hepatic vein. Eur J Gastroenterol Hepatol. 2012;24(2):186-94. doi 10.1097/MEG.0b013e32834dda64. [PubMed: 22081008].

31. Razavi H, Elkhoury AC, Elbasha E, Estes C, Pasini K, Poynard T, et al. Chronic hepatitis $C$ virus (HCV) disease burden and cost in the United States. Hepatology. 2013;57(6):2164-70. doi: 10.1002/hep.26218. [PubMed: 23280550].

32. Maylin S, Martinot-Peignoux M, Moucari R, Boyer N, Ripault MP, Cazals-Hatem D, et al. Eradication of hepatitis C virus in patients successfully treated for chronic hepatitis C. Gastroenterology. 2008;135(3):821-9. doi: 10.1053/j.gastro.2008.05.044. [PubMed: 18593587].

33. Butler J, Korda RJ, Watson KJ, Watson AR. The impact of chronic hepatitis B in Australia: Projecting mortality, morbidity and economic impact. Canberra: Australian Centre for Economic Research on Health. 2009.

34. Liu S, Cipriano LE, Holodniy M, Owens DK, Goldhaber-Fiebert JD. New protease inhibitors for the treatment of chronic hepatitis C: a cost-effectiveness analysis. Ann Intern Med. 2012;156(4):279-90. doi: 10.7326/0003-4819-156-4-201202210-00005. [PubMed: 22351713].

35. Wold Bank . PPP conversion factor, Private consumption Available from: http://data.worldbank.org/indicator/PA.NUS.PRVT.PP.

36. Elliott R, Payne K. Essentials of economic evaluation in healthcare Pharmaceutical Press; 2005.

37. Shillcutt SD, Walker DG, Goodman CA, Mills AJ. Cost effectiveness in low- and middle-income countries: a review of the debates surrounding decision rules. Pharmacoeconomics. 2009;27(11):903-17. doi: 10.2165/10899580-000000000-00000. [PubMed: 19888791].

38. Tantai N, Chaikledkaew U, Tanwandee T, Werayingyong P, Teerawattananon Y. A cost-utility analysis of drug treatments in patients with HBeAg-positive chronic hepatitis B in Thailand. BMC Health Serv Res. 2014;14:170. doi: 10.1186/1472-6963-14-170. [PubMed: 24731689].

39. Younossi ZM, Singer ME, Mir HM, Henry L, Hunt S. Impact of interferon free regimens on clinical and cost outcomes for chronic hepatitis C genotype 1 patients. I Hepatol. 2014;60(3):530-7. doi: 10.1016/j.jhep.2013.11.009. [PubMed: 24269472].

40. Dugum M, O'Shea R. Hepatitis C virus: here comes all-oral treatment. Cleve Clin J Med. 2014;81(3):159-72. doi: 10.3949/ccjm.81a.13155 [PubMed: 24591471].

41. Asselah T, Marcellin P. Second-wave IFN-based triple therapy for HCV genotype 1 infection: simeprevir, faldaprevir and sofosbuvir. Liver Int 2014;34 Suppl 1:60-8. doi: 10.1111/liv.12424. [PubMed: 24373080].

42. Younossi ZM, Stepanova M, Henry L, Gane E, Jacobson IM, Lawitz E, et al. Effects of sofosbuvir-based treatment, with and without interferon, on outcome and productivity of patients with chronic hepatitis C. Clinical Gastroenterol Hepatol. 2014;12(8):1349-59. 